\title{
An investigation of geographic object based image analysis (GEOBIA) for human settlement detection in South Africa
}

\author{
L. Ngcofe ${ }^{\mathrm{a},} *$, N. Mudau ${ }^{\mathrm{b}}$ \\ ${ }^{a}$ Department of Rural Development and Land Reform, Luncedo.Ngcofe@drdlr.gov.za \\ ${ }^{b}$ South African National Space Agency,nmudau@sansa.org.za
}

Keywords: GEOBIA, Human settlement mapping, Topographic update

\begin{abstract}
:
The changes to the landscape are constantly occurring both naturally and human induced. One of such changes is the human settlement expansion. The ability to map human settlements is vital for variety of studies including urban development planning and management. For this study human settlement detection is essential for topographic map update. The newly identified human settlements also serves as change detection area indicator for further update of other topographic features that are represented on the topographic map (such as roads etc.). The semi-automated human settlement detection has been conducted through geographic object based image analysis (GEOBIA) method using 2012 SPOT 5 imagery in the KwaZulu-Natal Province of South Africa. GEOBIA is relative new development of image processing and analysis of remote sensed imagery. It involves partitioning an imagery into discrete entities or segments from which meaningful image object, based on the spatial and spectral attributes can be generated. Through a multiresolution segmentation model implemented by the eCognition software, image segmentation was attained. This entailed evaluation of different segmentation parameter in order obtain suitable objects of interest. The following step was to determine appropriate variables obtained from image segmentation to classify the image. These include: layer values, geometry, position, texture, hierarchy and thematic attributes. The layer value option entails spectral statistics such as mean value and mean brightness for image reflectance bands together with capability of further applying band ratio combinations. Under the texture and geometry option also several alternatives are applicable (such as length/width under extend and asymmetry under shape properties). Under the level co-occurrence matrix (GLCM) the study explored the contrast textual measurements developed by Haralick et al., (1973). Other assessed variables are mean brightness, density, length/width and band ratios. The last step for GEOBIA was to determine suitable variables for the rule-set based classification. This resulted to $70.7 \%$ overall accuracy.
\end{abstract}

These results were further compared to the existing South African global human settlement layer (SA_GHSL) for the same study area which also used the same year 2012 SPOT 5 imagery. The SA_GHSL had an overall accuracy of $60 \%$. The GEOBIA presents an opportunity to apply semi-automated method to target areas of new settlement development more efficiently and with consistent repeatable manner. Thus assisting topographic update analyst to be drawn to more areas of new settlement development at an enhanced efficient rate. However the spectral variability of roof tops proved to be the most challenging obstacle towards of both the semi-automated settlement detection methods. 